\title{
NORMAL SUBGROUPS OF DOUBLY TRANSITIVE AUTOMORPHISM GROUPS OF CHAINS
}

BY

RICHARD N. BALL AND MANFRED DROSTE

Dedicated to W. Charles Holland on the occasion of his 50 th birthday

\begin{abstract}
We characterize the structure of the normal subgroup lattice of 2-transitive automorphism groups $A(\Omega)$ of infinite chains $(\Omega, \leqslant)$ by the structure of the Dedekind completion $(\bar{\Omega}, \leqslant)$ of the chain $(\Omega, \leqslant)$. As a consequence we obtain various group-theoretical results on the normal subgroups of $A(\Omega)$, including that any proper subnormal subgroup of $A(\Omega)$ is indeed normal and contained in a maximal proper normal subgroup of $A(\Omega)$, and that $A(\Omega)$ has precisely 5 normal subgroups if and only if the coterminality of the chain $(\Omega, \leqslant)$ is countable.
\end{abstract}

1. Introduction. An infinite linearly ordered set ("chain") $(\Omega, \leqslant)$ is called doubly homogeneous if its automorphsim group (i.e. the group of all order-preserving permutations) $A(\Omega)$ acts 2-transitively on it. Chains $(\Omega, \leqslant)$ of this type and their automorphism groups $A(\Omega)$ have been used for the construction of infinite simple torsion-free groups (Higman [12]) or, in the theory of lattice-ordered groups ( $l$ groups), in dealing with embeddings of arbitrary l-groups into simple divisible l-groups (Holland [13]) (for a variety of further results see Glass [10]).

$(\Omega, \leqslant)$ will designate a doubly homogeneous chain for the rest of this paper unless explicitly stated otherwise. We shall first characterize the structure of the normal subgroup lattice $\mathcal{N}(A(\Omega))$ of $A(\Omega)$ by the structure of the Dedekind completion $(\bar{\Omega}, \leqslant)$ of $(\Omega, \leqslant)$. In particular, automorphism groups of doubly homogeneous chains with isomorphic Dedekind completions have isomorphic normal subgroup lattices. Furthermore each proper subnormal subgroup of $A(\Omega)$ is indeed normal in $A(\Omega)$ and contained in a maximal proper normal subgroup of $A(\Omega)$. For $\alpha \in A(\Omega)$ let $(\alpha)$ denote the smallest normal subgroup of $A(\Omega)$ containing $\alpha$. We will show that whenever $\alpha, \beta \in A(\Omega)$ and $\alpha \in(\beta)$, then $\alpha$ is a product of 8 conjugates of $\beta$ and $\beta^{-1}$, and that there exists a sequence of exponents \pm 1 of $\beta$ which is independent of the particular choice of $\alpha, \beta \in A(\Omega)$. Similar results were established by Anderson [1] for groups of homeomorphisms of certain topological spaces and by Bertram [4] and Droste and Göbel [8] for permutation groups of unordered sets.

Received by the editors September 27, 1984

1980 Mathematics Subject Classification. Primary 06F15; Secondary 20B27, 20F38.

Key words and phrases. Order-preserving permutation, automorphism groups of chains, homogeneous linearly ordered sets, lattice-ordered groups, normal subgroup lattice, cofinality of a chain.

(C)1985 American Mathematical Society $0002-9947 / 85 \$ 1.00+\$ .25$ per page 
Obvious normal subgroups of $A(\Omega)$ are $R(\Omega)(L(\Omega))$, the group of all automorphisms with support bounded on the left (right, respectively), and $B(\Omega)=R(\Omega) \cap$ $L(\Omega)$. According to Higman [12], $B(\Omega)$ is always simple and contained in every nontrivial normal subgroup of $A(\Omega)$. Holland [13] and Lloyd [16] showed that if the cofinality $\operatorname{cof}(\Omega)$ (coinitiality $\operatorname{coi}(\Omega)$ ) of $\Omega$ is countable, then the group $R(\Omega) / B(\Omega)$ $(L(\Omega) / B(\Omega)$ ) is simple. Contradicting [17] the first author [2] showed by example that in general the assumption $\operatorname{cof}(\Omega)=\boldsymbol{\aleph}_{0}$ is necessary. Here, as a consequence of our characterization of the lattice $\mathscr{N}(A(\Omega))$, we shall show that indeed for any doubly homogeneous chain $\Omega$ with $\operatorname{cof}(\Omega) \neq \boldsymbol{\aleph}_{0}$ the partially ordered set $\{N \mid B(\Omega) \subsetneq N \triangleleft R(\Omega)\}$ contains a smallest element different from $R(\Omega)$; hence it is isomorphic to the lattice of all ideals of the partially ordered subset $\mathscr{R}_{1}=\{(\alpha) \mid \alpha \in$ $R(\Omega) \backslash B(\Omega)\}$. By a statement about $(\bar{\Omega}, \leqslant)$ we will characterize when $\left(\mathscr{R}_{1}, \subseteq\right)$ is a Boolean algebra; in this case $\mathscr{R}_{1}$ is isomorphic to a Boolean algebra $\mathscr{A}(\kappa)$ depending only on $\kappa=\operatorname{cof}(\Omega)$.

Finally, considering $A(\Omega)$ as an l-group again, we get that each proper normal subgroup of $A(\Omega)$ is an intersection of minimal prime $l$-subgroups of $A(\Omega)$.

For further results on the structure of $\mathscr{N}(A(\Omega))$ see [6, 7] and Droste and Shelah [9].

This paper contains parts of the dissertation thesis [2 and 6] of the authors, written under the supervision of Professor W. C. Holland, Bowling Green, and Professor R. Göbel, Essen, respectively. In [2], the results of this paper were first established for a certain class of doubly homogeneous chains $(\Omega, \leqslant)$ with $\operatorname{cof}(\Omega)=$ $\boldsymbol{\aleph}_{1}$; in [6] then the general theory was developed. The authors would like to thank Professors Holland and Göbel for their encouragement.

2. Notation and remarks. For background results on our topic we refer the reader to Glass [10]. $A \dot{\cup} B$ and $\dot{\cup} A_{i}$ denote disjoint unions. For a mapping $f$ we let $a^{f}$ denote its value at $a$ and $\left.f\right|_{A}$ its restriction to $A$. If $A_{i}(i \in I)$ are pairwise disjoint sets and $\alpha_{i}: A_{i} \rightarrow M_{i}$ maps, we denote by $\alpha=\bigoplus_{i \in I} \alpha_{i}$ the map from $\dot{\cup}_{i \in I} A_{i}$ into $\cup_{i \in I} M_{i}$ defined by $\left.\alpha\right|_{A_{i}}=\alpha_{i}(i \in I)$. As usual, cardinal numbers are identified with the least ordinals of the same cardinality.

Let $(\Omega, \leqslant)$ be a dense unbounded chain. Then $(\bar{\Omega}, \leqslant)$ denotes the Dedekind completion of $(\Omega, \leqslant)$, and $(\hat{\Omega}, \leqslant)$ denotes the absolute completion of $(\Omega, \leqslant)$. That is, $\hat{\Omega}=\bar{\Omega} \dot{\cup}\{-\infty, \infty\}$, where $-\infty<x<\infty$ for each $x \in \bar{\Omega}$. Note that each subset of $\Omega$ has a supremum and an infimum in $\hat{\Omega}$. If $a, b \in \hat{\Omega}$ satisfy $a<b$, let $[a, b]=\{z \in$ $\hat{\Omega} \mid a \leqslant z \leqslant b\}$. For each $a \in \bar{\Omega} \cup\{\infty\}$ let the cofinality of $a$ be

$$
\operatorname{cof}(a)=\inf \{|A| \mid a \notin A \subseteq \bar{\Omega}, a=\sup A\},
$$

and for $b \in \bar{\Omega} \cup\{-\infty\}$ let the coinitiality of $b$ be

$$
\operatorname{coi}(b)=\inf \{|B| \mid b \notin B \subseteq \bar{\Omega}, b=\inf B\} .
$$

The cofinality of $\Omega$ is $\operatorname{cof}(\Omega)=\operatorname{cof}(\infty)$, the coinitiality of $\Omega$ is $\operatorname{coi}(\Omega)=\operatorname{coi}(-\infty)$, and the coterminality of $\Omega$ is $\cot (\Omega)=\operatorname{cof}(\Omega)$ in case $\operatorname{cof}(\Omega)=\operatorname{coi}(\Omega)$. Now let $(\Omega, \leqslant)$ be an infinite chain and $k \in \mathrm{N} . A(\Omega)$ is $k$-transitive if whenever $A, B \subseteq \Omega$ with $|A|=|B|=k$, there exists $\alpha \in A(\Omega)$ with $A^{\alpha}=B$. The following remarks are well known. 
REMARK 2.1. An infinite chain $(\Omega, \leqslant)$ is called doubly homogeneous if one of the following three pairwise equivalent conditions is satisfied:

(1) $A(\Omega)$ is $k$-transitive for some $k \in \mathbf{N}$ with $k \geqslant 2$.

(2) $A(\Omega)$ is $k$-transitive for any $k \in \mathbf{N}$ with $k \geqslant 2$.

(3) $\Omega$ is unbounded, and any two intervals $[a, b] \cap \Omega,[c, d] \cap \Omega(a, b, c, d \in \Omega$, $a<b, c<d)$ in $\Omega$ are order-isomorphic.

REMARK 2.2. If $\left\{a_{i} \mid i \in \mathbf{Z}\right\}$ and $\left\{b_{i} \mid i \in \mathbf{Z}\right\}$ are two subsets of the doubly homogeneous chain $\Omega$ such that $a_{i}<a_{i+1}$ and $b_{i}<b_{i+1}$ for all $i \in \mathbf{Z}$ and such that the two subsets have the same supremum and the same infimum (in $\bar{\Omega}$ ), then there is some $\alpha \in A(\Omega)$ such that $a_{i}^{\alpha}=b_{i}$ for all $i \in \mathbf{Z}$.

REMARK 2.3. Let $\Omega$ be a doubly homogeneous chain. Then $\Omega$ is dense and unbounded. For any $a \in \bar{\Omega}$, the set $\{x \in \bar{\Omega} \mid \operatorname{cof}(x)=\operatorname{cof}(a), \operatorname{coi}(x)=\operatorname{coi}(a)\}$ is dense in $\bar{\Omega}$. In particular, the sets $\left\{x \in \bar{\Omega} \mid \operatorname{cof}(x)=\boldsymbol{\aleph}_{0}\right\}$ and $\left\{x \in \bar{\Omega} \operatorname{coi}(x)=\boldsymbol{\aleph}_{0}\right\}$ are dense in $\bar{\Omega}$.

We let id denote the identity map of $\Omega$. $A(\Omega)$ becomes an l-group if we put $a^{\alpha \vee \beta}=\max \left\{a^{\alpha}, a^{\beta}\right\}, a^{\alpha \wedge \beta}=\min \left\{a^{\alpha}, a^{\beta}\right\}$ for all $\alpha, \beta \in A(\Omega), a \in \Omega$. If $\alpha \in A(\Omega)$, let $\alpha^{+}=\alpha \vee \mathrm{id}, \alpha^{-}=\alpha \wedge \mathrm{id}$, and $|\alpha|=\alpha \vee \alpha^{-1}$. If $G$ is any l-group, a convex normal $l$-subgroup of $G$ is also called an $l$-ideal. For $1<g \in G$ let $g^{\perp}=\{x \in G|| x \mid$ $\wedge g=1\}$, and put $C^{\perp}=\bigcap\left\{c^{\perp} \mid c \in C\right\}$ for any $C \subseteq G$. A polar of $G$ is a subset of $D$ for which $D=D^{\perp \perp} ; D$ is termed principal if $D=d^{\perp \perp}$ for some $d \in D$.

3. Characterization of $\mathscr{N}(A(\Omega))$. The goal of this section is to characterize the structure of the lattice $(\mathscr{N}(A(\Omega)), \subseteq)$ by the structure of the Dedekind completion $(\bar{\Omega}, \leqslant)$ of $(\Omega, \leqslant)$. Let us first summarize some properties of $\mathscr{N}(A(\Omega))$ already known which will be important to us.

THEOREM 3.1. Let $\Omega$ be a doubly homogeneous chain. Then:

(a) Every normal subgroup of $A(\Omega)$ is an l-ideal of $A(\Omega)$.

(b) Every normal subgroup of $R(\Omega)(L(\Omega), B(\Omega))$ is also normal in $A(\Omega)$.

(c) $B(\Omega)$ is the smallest normal subgroup of $A(\Omega)$.

(d) $A(\Omega)=L(\Omega) \cdot R(\Omega)$.

(e) If $\operatorname{cof}(\Omega)=\boldsymbol{\aleph}_{0}$, then $R(\Omega) / B(\Omega)$ is simple.

(f) If coi $(\Omega)=\aleph_{0}$, then $L(\Omega) / B(\Omega)$ is simple.

(g) If $\cot (\Omega)=\boldsymbol{\aleph}_{0}$, then $B(\Omega), R(\Omega)$ and $L(\Omega)$ are the only nontrivial proper normal and subnormal subgroups of $A(\Omega)$.

Here (c) is due to Higman [12], whereas the other statements are slight generalizations of results due to Holland [13] and Lloyd [16] (cf. $\$ 2.3$ of [10]). We will now examine the structure of $(\mathcal{N}, \subseteq)$ for arbitrary cofinality and coinitiality of $\Omega$.

Definition 3.2. For every group $G$ and $\alpha \in G$ let $(\alpha)=\left\langle\alpha^{G}\right\rangle=\bigcap\{N \mid \alpha \in N \triangleleft G\}$ be the normal subgroup of $G$ generated by $\alpha$. We put $\mathscr{N}_{1}(G)=\{(\alpha) \mid \alpha \in G\}$.

We abbreviate $\mathscr{N}_{1}(A(\Omega))$ by writing simply $\mathscr{N}_{1}$. First we show that the structure of $(\mathscr{N}, \subseteq)$ is completely determined by the structure of $\left(\mathscr{N}_{1}, \subseteq\right)$ and vice-versa.

Proposition 3.3. $\left(\mathscr{N}_{1}, \subseteq\right)$ is a join-semilattice, i.e. $(\alpha) \cdot(\beta) \in \mathscr{N}_{1}$ for all $\alpha, \beta \in$ $A(\Omega)$, and thus coincides with the set of all those elements of $\mathscr{N}$ which are finitely 
generated as normal subgroups. $(\mathcal{N}, \subseteq)$ is a complete algebraic Brouwerian lattice with $\mathscr{N}_{1}$ as its set of compact elements. In particular, $(\mathscr{N}, \subseteq)$ is distributive and isomorphic to the set of all ideals of $\left(\mathscr{N}_{1}, \subseteq\right)$.

Proof. Because of Theorem 3.1(a) we have to prove a version of our claims where " $l$-ideal" is substituted everywhere for "normal subgroup." But, indeed, this $l$-ideal version holds for every l-group $G$ (cf. [5, pp. 187, 304, 306]).

Now we turn to an examination of the partially ordered set $\left(\mathscr{N}_{1}, \subseteq\right)$. We first examine statements of the form " $\alpha \in(\beta)$ " for $\alpha, \beta \in A(\Omega)$ in case $\Omega$ has countable coterminality. Here we make use of the fact that $A(\Omega)$ is an l-group. For the techniques used in the proofs of 3.4-3.10 we refer the reader to $\$ 2.2$ of Glass [10]. Given $\alpha, \beta \in A(\Omega), \alpha^{\beta}$ denotes $\beta^{-1} \alpha \beta$.

Lemma 3.4 (Holland [13]). Let $\alpha, \beta \in A(\Omega)$.

(a) Assume that for each $a \in \Omega, a<a^{\alpha}\left(a=a^{\alpha}, a^{\alpha}<a\right)$ implies $a<a^{\beta}(a=$ $a^{\beta}, a^{\beta}<a$, respectively). Then there exists $a \gamma \in A(\Omega)$ with $\alpha=\beta^{\gamma}$.

(b) If $\alpha$ and $\beta$ are conjugate and $\beta \in R(\Omega)(L(\Omega), B(\Omega))$ then there is also a conjugator $\gamma \in R(\Omega)(L(\Omega), B(\Omega))$ with $\alpha=\beta^{\gamma}$.

LEMma 3.5. For every $\alpha \in A(\Omega)$ there are $\gamma_{1}, \gamma_{2} \in A(\Omega)$ with $|\alpha|=\alpha^{\gamma_{1}} \cdot\left(\alpha^{-1}\right)^{\gamma_{2}}$.

Proof. First note $\alpha=\alpha^{+} \cdot \alpha^{-}=\alpha^{-} \cdot \alpha^{+}$. Let $\alpha_{1}=\left(\alpha^{+}\right)^{2} \cdot \alpha^{-}$and $\alpha_{2}=\alpha^{+} \cdot\left(\alpha^{-}\right)^{2}$. Hence by Lemma 3.4(a) there are $\gamma_{i} \in A(\Omega)$ with $\alpha_{i}=\alpha^{\gamma_{i}}(i=1,2)$. Thus

$$
|\alpha|=\alpha^{+} \cdot\left(\alpha^{-}\right)^{-1}=\alpha_{1} \cdot \alpha_{2}^{-1}=\alpha^{\gamma_{1}} \cdot\left(\alpha^{-1}\right)^{\gamma_{2}} .
$$

Lemma 3.6 (Holland [13]). Assume $\cot (\Omega)=\aleph_{0}$ and let $\alpha, \beta \in A(\Omega)$ with id $\leqslant \alpha$, id $<\beta$. Furthermore assume that whenever $\beta \in R(\Omega) \backslash B(\Omega)(\beta \in L(\Omega) \backslash B(\Omega)$, $\beta \in B(\Omega)$ ), then $\alpha \in R(\Omega)(\alpha \in L(\Omega), \alpha \in B(\Omega)$, respectively). Then there are $\gamma_{1}, \gamma_{2} \in A(\Omega)$ with $\alpha \leqslant \beta^{\gamma_{1}} \vee \beta^{\gamma_{2}}$.

We now come to the first important new result of this section.

LEMMA 3.7. Let $\Omega$ be a doubly homogeneous chain with countable coterminality, and $\alpha, \beta \in A(\Omega)$ such that $\beta \neq \mathrm{id}$. The following are equivalent.

(1) $(\alpha) \subseteq(\beta)$.

(2) If $\beta \in R(\Omega) \backslash B(\Omega)(\beta \in L(\Omega) \backslash B(\Omega), \beta \in B(\Omega))$, then $\alpha \in R(\Omega)(\alpha \in L(\Omega)$, $\alpha \in B(\Omega)$, respectively).

(3) There are $\gamma_{i}, \gamma_{i}^{\prime} \in A(\Omega)(i=1,2,3,4)$ such that $\alpha=\prod_{i=1}^{4}\left(\beta^{\gamma_{i}} \cdot\left(\beta^{-1}\right)^{\gamma_{i}^{\prime}}\right)$.

(4) There are $\gamma_{i}, \gamma_{i}^{\prime} \in(\beta)(i=1,2,3,4)$ such that $\alpha=\prod_{i=1}^{4}\left(\beta^{\gamma_{i}} \cdot\left(\beta^{-1}\right)^{\gamma_{i}^{\prime}}\right)$.

Proof. (4) $\rightarrow$ (3) $\rightarrow$ (1) $\rightarrow$ (2): Trivial.

$(2) \rightarrow$ (3): According to Lemma 3.6 there are $\gamma_{1}, \gamma_{2} \in A(\Omega)$ with $|\alpha|^{2} \leqslant|\beta|^{\gamma_{1}} \vee$ $|\beta|^{\gamma_{2}} \leqslant|\beta|^{\gamma_{1}} \cdot|\beta|^{\gamma_{2}}=\delta$. Note that id $\leqslant \alpha \cdot|\alpha| \leqslant \delta$ and $|\alpha| \leqslant \delta$, thus id $\leqslant \delta \leqslant\{\alpha$. $|\alpha| \cdot \delta,|\alpha| \cdot \delta\} \leqslant \delta^{2}$. By Lemma 3.4(a) we obtain $\gamma_{3}, \gamma_{4} \in A(\Omega)$ with $\alpha \cdot|\alpha| \cdot \delta=\delta^{\gamma_{3}}$ and $|\alpha| \cdot \delta=\delta^{\gamma_{4}}$. Hence

$$
\boldsymbol{\alpha}=\boldsymbol{\delta}^{\gamma_{3}} \cdot\left(\boldsymbol{\delta}^{\gamma_{4}}\right)^{-1}=|\beta|^{\gamma_{1} \cdot \gamma_{3}} \cdot|\beta|^{\gamma_{2} \cdot \gamma_{3}} \cdot\left(|\beta|^{-1}\right)^{\gamma_{2} \cdot \gamma_{4}} \cdot\left(|\beta|^{-1}\right)^{\gamma_{1} \cdot \gamma_{4}}
$$


By Lemma 3.5 there are $\gamma_{5}, \gamma_{6} \in A(\Omega)$ with $|\beta|=\beta^{\gamma_{5}} \cdot\left(\beta^{-1}\right)^{\gamma_{6}},|\beta|^{-1}=\beta^{\gamma_{6}} \cdot\left(\beta^{-1}\right)^{\gamma_{5}}$. This implies (3).

(3) $\rightarrow(4)$ : If $\beta \notin R(\Omega) \cup L(\Omega)$, then $(\beta)=A(\Omega)$ by what we have already proved. Now assume, e.g., $\beta \in R(\Omega)$ and let $i \in\{1,2,3,4\}$. By Lemma 3.4(b) there exists a $\bar{\gamma}_{i} \in R(\Omega)$ with $\beta^{\gamma_{i}}=\beta^{\gamma_{i}}$; furthermore, $\bar{\gamma}_{i} \in B(\Omega)$ if $\beta \in B(\Omega)$. Now if $B \in R(\Omega) \backslash B(\Omega)$ or $\beta \in B(\Omega)$, we have $\bar{\gamma}_{i} \in(\beta)$ according to $(2) \rightarrow(1)$.

Note that Lemma $3.7((1) \Leftrightarrow(2))$ coincides with Theorem 3.1(g). Now we want to generalize Lemma 3.7 to chains $\Omega$ of arbitrary cofinality and coinitiality, and we wish to characterize statements of the form " $\alpha \in(\beta)$ " for $\alpha, \beta \in A(\Omega)$ by statements about $(\bar{\Omega}, \leqslant)$. It will be crucial that in Lemma 3.7(3) we have obtained an order for the exponents \pm 1 of $\beta$ which is independent of the special choice of $\alpha, \beta \in A(\Omega)$. We first need some preparation.

Notation 3.8. Let $\bar{\Omega}$ be the Dedekind completion and $\hat{\Omega}=\bar{\Omega} \dot{\cup}\{-\infty, \infty\}$ the absolute completion of $\Omega$. If $\alpha \in A(\Omega)$, we put $F(\alpha)=\left\{a \in \hat{\Omega} \mid a^{\hat{\alpha}}=a\right\}$, where $\hat{\alpha}$ denotes the uniquely determined extension of $\alpha$ to an automorphism of $\hat{\Omega}$. We always have $-\infty, \infty \in F(\alpha)$. Let $\mathscr{F}=\mathscr{F}(\Omega)=\{F(\alpha) \mid \alpha \in A(\Omega)\}$. If $a, b \in \hat{\Omega}$ satisfy $a<b$, we always consider $(a, b)=\{x \in \bar{\Omega} \mid a<x<b\}$ as the Dedekind completion and $[a, b]=(a, b) \dot{\cup}\{a, b\}$ as the absolute completion of $\Omega_{a, b}=\Omega \cap(a, b)$. By Remark 2.1, $\Omega_{a, b}$ is a doubly homogeneous chain. Hence for $\alpha \in A\left(\Omega_{a, b}\right)$ we have $a, b \in F(\alpha) \subseteq[a, b]$. For every subset $A \subseteq \hat{\Omega}$ let $A^{c}=\hat{\Omega} \backslash A$.

The following two remarks are well known and very important to us.

REMARK 3.9. F is closed under finite intersections.

Proof. Let $\alpha, \beta \in A(\Omega)$. Put $\gamma=|\alpha| \cdot|\beta| \in A(\Omega)$. Then $F(\alpha) \cap F(\beta)=F(\gamma) \in$ $\mathscr{F}$.

In fact, $\mathscr{F}$ is closed under even countably infinite intersections, as we will see in Proposition 3.16(c), but we will not need this generalization.

REMARK 3.10. Let $A \subseteq \hat{\Omega}$. The following are equivalent:

(1) $A \in \mathscr{F}$.

(2) $A^{c} \subseteq \bar{\Omega}$ and $A^{c}$ is a disjoint union of open intervals of countable coterminality.

Proof. (1) $\rightarrow$ (2): Let $\alpha \in A(\Omega)$ satisfy $A=F(\alpha)=F(|\alpha|)$. Then for each $a \in A^{c}$ $\cap \Omega$, the set $I_{a}=\bigcup_{n \in \mathrm{N}}\left(a^{|\alpha|^{-n}}, a^{|\alpha|^{n}}\right) \subseteq \bar{\Omega}$ is an open interval of countable coterminality, $A^{c}=\bigcup\left\{I_{a} \mid a \in A^{c} \cap \Omega\right\}$, and for any $a, b \in A^{c} \cap \Omega$, either $I_{a}=I_{b}$ or $I_{a} \cap I_{b}=$ $\varnothing$.

(2) $\rightarrow$ (1): Let $(a, b) \subseteq A^{c}$ be an open interval with $a, b \in A$ and $\cot ((a, b))=\boldsymbol{\aleph}_{0}$. Then $\Omega_{a}=(a, b) \cap \Omega$ is doubly homogeneous. Choose an unbounded subset $\left\{a_{i} \mid i\right.$ $\in \mathbf{Z}\} \subseteq \Omega_{a}$ with $a_{i}<a_{i+1}$ for each $i \in \mathbf{Z}$, and, by Remark 2.2, an $\alpha_{a} \in A\left(\Omega_{a}\right)$ with $a_{i}^{\alpha_{a}}=a_{i+1}$. Then $F\left(\alpha_{a}\right)=\{a, b\}$. If we define $\alpha \in A(\Omega)$ by $\left.\alpha\right|_{\Omega_{a}}=\alpha_{a}$ for each such interval $(a, b)$, we obtain $A=F(\alpha) \in \mathscr{F}$.

Observe that Remark 3.10 characterizes the statement " $A \in \mathscr{F}$ " completely within $(\hat{\Omega}, \leqslant)$, without referring to $A(\Omega)$.

Definition 3.11. For each subset $A \subseteq \hat{\Omega}$ we put

$S(A)=\{a \in A \mid$ for no $b \in \bar{\Omega}$ with $b<a$ do we have $[b, a] \subseteq A$, and for no $c \in \bar{\Omega}$ with $a<c$ do we have $[a, c] \subseteq A\}$,

$R(A)=\{a \in A \mid$ there exists $c \in \bar{\Omega}$ with $a<c$ and $[a, c] \subseteq A$, but no $b \in \bar{\Omega}$ with $b<a$ and $[b, a] \subseteq A\}$, 
$L(A)=\{a \in A \mid$ there exists $b \in \bar{\Omega}$ with $b<a$ and $[b, a] \subseteq A$, but no $c \in \bar{\Omega}$ with $a<c$ and $[a, c] \subseteq A\}$,

$I(A)=\{a \in A \mid$ there exist $b, c \in \bar{\Omega}$ with $b<a<c$ and $[b, c] \subseteq A\}$.

If $A=F(\alpha)$ for some $\alpha \in A(\Omega)$, we also put $S(\alpha)=S(A), R(\alpha)=R(A), L(\alpha)$ $=L(A)$ and $I(\alpha)=I(A)$.

We always have $A=S(A) \dot{\cup} R(A) \dot{\cup} L(A) \dot{\cup} I(A)$, and $I(A)$ is the interior of $A$. The set $\{I(A) \mid A \in \mathscr{F}\}$ coincides with the set of all regular open subsets of $\bar{\Omega}$. If $\alpha \in A(\Omega)$ and $x \in \hat{\Omega}$, we have $x \in R(\alpha)(x \in L(\alpha))$ iff a whole interval on the right (left), but not on the left (right) of $x$ is fixed by $\alpha$. We always have $-\infty \in R(\alpha) \cup S(\alpha)$ and $\infty \in L(\alpha) \cup S(\alpha)$, and, finally, $\alpha \in L(\Omega)(\alpha \in R(\Omega), \alpha \in B(\Omega), \alpha=$ id) iff $\infty \in L(\alpha)(-\infty \in R(\alpha), \infty \in L(\alpha)$ and $-\infty \in R(\alpha), F(\alpha)=\hat{\Omega}$, respectively).

We come now to our generalization of Lemma 3.7.

THEOREM 3.12. Let $\Omega$ be a doubly homogeneous chain, and let $\alpha, \beta \in A(\Omega)$ and $A=F(\alpha), B=F(\beta)$. The following are equivalent.

(1) $(\alpha) \subseteq(\beta)$,

(2) There exists an $F \in \mathscr{F}$ with $F \subseteq A \cap B$ and the following properties:

(i) $I(B) \cap F \subseteq I(A)$.

(ii) $R(B) \cap F \subseteq R(A) \cup I(A)$,

(iii) $L(B) \cap F \subseteq L(A) \cup I(A)$,

(iv) whenever $a, b \in F$ satisfy $a<b$ and $[a, b] \subseteq B$, then $[a, b] \subseteq A$.

Here, (i)-(iii) may be replaced by

(i') $S(A) \cap F \subseteq S(B)$,

(ii') $R(A) \cap F \subseteq R(B) \cup S(B)$,

(iii') $L(A) \cap F \subseteq L(B) \cup S(B)$.

(3) There are $\gamma_{i}, \gamma_{i}^{\prime} \in(\beta) \subseteq A(\Omega)(i=1,2,3,4)$ with $\alpha=\prod_{i=1}^{4}\left(\beta^{\gamma_{i}} \cdot\left(\beta^{-1}\right)^{\gamma_{i}^{\prime}}\right)$.

Proof. (1) $\rightarrow$ (2): There exist $n \in \mathbf{N}$ and $\gamma_{i} \in A(\Omega), \beta_{i} \in\left\{\beta, \beta^{-1}\right\}(i=1, \ldots, n)$ such that $\alpha=\prod_{i=1}^{n} \beta_{i}^{\gamma_{i}}$. Let $F=B \cap \bigcap_{i=1}^{n} F\left(\gamma_{i}\right)$. By Remark 3.9 we have $F \in \mathscr{F}$ and $F \subseteq A \cap B$. We first show $(R(B) \cup I(B)) \cap F \subseteq R(A) \cup I(A)$, thus in particular (2)(ii). Let $a \in(R(B) \cup I(B)) \cap F$. Then there is some $c \in \bar{\Omega}$ with $a<c$ and $[a, c] \subseteq B$, and since $a \in \bigcap_{i=1}^{n} F\left(\gamma_{i}\right)$, we have $x^{\gamma_{i}}, x^{\gamma_{i}^{-1}} \in(a, c)$ for all $x \in(a, d) \cap \Omega$ and $i=1, \ldots, n$, where $d$ is the least among $\left\{c^{\gamma_{i}^{ \pm 1}} \mid i=1, \ldots, n\right\}$. Hence $[a, d) \subseteq A$ and $a \in R(A) \cup I(A)$.

Analogously, we obtain $(L(B) \cup I(B)) \cap F \subseteq L(A) \cup I(A)$ and thus (2)(iii).

This implies

$$
I(B) \cap F=(L(B) \cup I(B)) \cap(R(B) \cup I(B)) \cap F \subseteq I(A),
$$

hence (2)(i). Now let $a, b \in F$ with $a<b$ and $[a, b] \subseteq B$. Then $a, b \in F\left(\gamma_{i}\right)$, hence $([a, b] \cap \Omega)^{\gamma_{i}}=[a, b] \cap \Omega$ for $i=1, \ldots, n$. Thus $[a, b] \subseteq F(\alpha)=A$, proving (2)(i)-(iv). The equivalence of (i)-(iii) and (i')-(iii') is obvious.

(2) $\rightarrow$ (3): If $F=\hat{\Omega}$, we have $A=B=\hat{\Omega}$, thus $\alpha=\beta=$ id and the assertion is trivial. Now assume $F \neq \hat{\Omega}$. By Remark 3.10 there exists a nonempty index set $I$ with $F^{c}=\dot{U}_{i \in I}\left(a_{i}, b_{i}\right), a_{i}, b_{i} \in F$, and $\cot \left(\left(a_{i}, b_{i}\right)\right)=\aleph_{0}$ for each $i \in I$. For each $i \in I$, let $\Omega_{i}=\Omega \cap\left(a_{i}, b_{i}\right), \alpha_{i}=\left.\alpha\right|_{\Omega_{i}}$ and $\beta_{i}=\left.\beta\right|_{\Omega_{i}}$. Then $\Omega_{i}$ is a doubly homogeneous 
chain with countable coterminality and $\alpha_{i}, \beta_{i} \in A\left(\Omega_{i}\right)$ because $a_{i}, b_{i} \in F \subseteq A \cap B$. We claim that $\beta_{i} \in R\left(\Omega_{i}\right)$ implies $\alpha_{i} \in R\left(\Omega_{i}\right)$, for if not then $a_{i} \in L(A) \cup S(A)$, hence $a_{i} \in L(B) \cup S(B)$ by our assumption, contrary to $\beta_{i} \in R\left(\Omega_{i}\right)$. Similarly $\beta_{i} \in L\left(\Omega_{i}\right)$ implies $\alpha_{i} \in L\left(\Omega_{i}\right)$. Hence $\beta_{i} \in B\left(\Omega_{i}\right)$ implies $\alpha_{i} \in B\left(\Omega_{i}\right)$. Finally, if $\beta_{i}=\left.\mathrm{id}\right|_{\Omega_{i}}$, then $\left[a_{i}, b_{i}\right] \subseteq B$, thus $[a, b] \subseteq A$ and $\alpha_{i}=\left.\mathrm{id}\right|_{\Omega_{i}}$. Now by Lemma 3.7 (applied twice) there are

$$
\gamma_{i j}, \gamma_{i j}^{\prime} \in\left(\beta_{i}^{A\left(\Omega_{i}\right)} \cdot\left(\beta_{i}^{-1}\right)^{A\left(\Omega_{i}\right)}\right)^{4} \subseteq A\left(\Omega_{i}\right) \quad(j=1,2,3,4)
$$

with $\alpha_{i}=\prod_{j=1}^{4}\left(\beta_{i}^{\gamma_{i j}} \cdot\left(\beta_{i}^{-1}\right)^{\gamma_{i j}^{\prime}}\right)$. Put $\gamma_{j}=\left.\oplus_{i \in I} \gamma_{i j} \oplus \mathrm{id}\right|_{F \cap \Omega} \in A(\Omega), \gamma_{j}^{\prime}=\oplus_{i \in I} \gamma_{i j}^{\prime}$ $\left.\oplus \mathrm{id}\right|_{F \cap \Omega} \in A(\Omega)(j=1,2,3,4)$. Since $F \subseteq A \cap B$, we have

$$
\alpha=\left.\bigoplus_{i=1} \alpha_{i} \oplus \mathrm{id}\right|_{F \cap \Omega} \text { and } \beta=\left.\bigoplus_{i \in I} \beta_{i} \oplus \mathrm{id}\right|_{F \cap \Omega},
$$

hence $\alpha=\prod_{j=1}^{4}\left(\beta^{\gamma_{j}} \cdot\left(\beta^{-1}\right)^{\gamma_{j}^{\prime}}\right)$. Similarly we obtain $\gamma_{j}, \gamma_{j}^{\prime} \in\left(\beta^{A(\Omega)} \cdot\left(\beta^{-1}\right)^{A(\Omega)}\right)^{4} \subseteq$ ( $\beta$ ) for $j=1,2,3,4$.

(3) $\rightarrow$ (1): Trivial.

As an immediate consequence of Theorem 3.12, we note that there is a single formula $\varphi(x, y)$ in the first order language of predicate calculus for group theory such that whenever $\alpha, \beta \in A(\Omega)$, then $\varphi[\alpha, \beta]$ holds iff $(\alpha) \subseteq(\beta)$. We now generalize Theorem 3.1(b).

COROllary 3.13. Let $\Omega$ be a doubly homogeneous chain. Then any subnormal subgroup of $A(\Omega)$ is normal in $A(\Omega)$.

Proof. Let $N \triangleleft M \triangleleft A(\Omega), \beta \in N, \gamma \in A(\Omega)$ and $\alpha=\beta^{\gamma}$. By Theorem 3.12, $\alpha=\prod_{i=1}^{4}\left(\beta^{\gamma_{i}} \cdot\left(\beta^{-1}\right)^{\gamma_{i}^{\prime}}\right)$ for some $\gamma_{i}, \gamma_{i}^{\prime} \in(\beta) \subseteq M(i=1,2,3,4)$. Hence $\alpha \in N$, which shows $N \triangleleft A(\Omega)$.

For later purposes (see Theorem 5.5) we note here:

Corollary 3.14. Let $\Omega$ be a doubly homogeneous chain and $\alpha, \beta \in A(\Omega)$. Then $I(\beta) \subseteq I(\alpha)$ implies $(\alpha) \subseteq(\beta)$. In particular, $I(\alpha)=I(\beta)$ implies $(\alpha)=(\beta)$.

Proof. Apply Theorem 3.12 with $F=A \cap B$.

Next we want to simplify condition (2) of Theorem 3.12.

Definition 3.15. Let $A \subseteq \hat{\Omega}$. We put $\lim (A)=\{a \in \bar{\Omega} \dot{\cup}\{\infty\} \mid a=\sup \{x \in A \mid x$ $<a\}\}$ and $\lim _{\leftarrow}(A)=\{a \in \bar{\Omega} \dot{\cup}\{-\infty\} \mid \vec{a}=\inf \{x \in A \mid a<x\}\}$. We call $A$ closed upwards (downwards) if $\lim _{\rightarrow}(A) \subseteq A \quad\left(\lim _{\leftarrow}(A) \subseteq(A)\right)$. Let $\lim (A)=\lim _{\rightarrow}(A)$ $\cup \lim (A)$, the set of all accumulation points of $A$. We say that $A$ is cofinal to $a \in \overleftarrow{\bar{\Omega}} \cup\{\infty\}$ if $a \in \lim _{\rightarrow}(A)$, and coinitial to $b \in \bar{\Omega} \dot{\cup}\{-\infty\}$ if $b \in \underset{\leftarrow}{\lim }(A) ; A$ is cofinal (coinitial) if $A$ is cofinal to $\infty$ (coinitial to $-\infty$ ).

Now we list several important properties for subsets of $\hat{\Omega}$ which will be used very often in the following.

Proposition 3.16. Let $A \subseteq \hat{\Omega}$.

(a) $A$ is closed iff $A$ is closed upwards and downwards, i.e. iff $\lim (A) \subseteq A$.

(b) $A \in \mathscr{F}$ iff $-\infty, \infty \in A$ and $A$ is closed and cofinal (coinitial) to each $a \in$ $A \backslash\{-\infty\}(a \in A \backslash\{\infty\})$ with $\operatorname{cof}(a) \neq \boldsymbol{\aleph}_{0}\left(\operatorname{coi}(a) \neq \boldsymbol{\aleph}_{0}\right)$ 
(c) Let $A_{i} \subseteq \hat{\Omega}(i \in I)$ and $A=\bigcap_{i \in I} A_{i}$.

(i) If all $A_{i}(i \in I)$ are closed upwards (downwards), then so is $A$.

(ii) Let I be countable. If all $A_{i}, i \in I$, are closed upwards (downwards) and cofinal to $a \in \bar{\Omega} \cup\{\infty\}$ (coinitial to $a \in \bar{\Omega} \cup\{-\infty\}$ ), where $\operatorname{cof}(a) \neq \boldsymbol{\aleph}_{0}\left(\operatorname{coi}(a) \neq \boldsymbol{\aleph}_{0}\right)$, then $A$ is closed upwards (downwards) and cofinal (coinitial) to a.

(iii) Let $I$ be countable and $A_{i} \in \mathscr{F}$ for each $i \in I$. Then $A \in \mathscr{F}$.

(d) Let $n \in \mathbf{N}$ and $A=\bigcup_{i=1}^{n} A_{i}$ with $A_{i} \subseteq \hat{\Omega}(i=1, \ldots, n)$.

(i) We have $\lim (A)=\bigcup_{i=1}^{n} \lim \left(A_{i}\right)$ and $\lim (A)=\bigcup_{i=1}^{n} \lim \left(A_{i}\right)$. In particular, if $\lim \left(A_{i}\right) \subseteq A\left(\overrightarrow{\lim }\left(A_{i}\right) \subseteq A\right)$ for all $i=1, \ldots, n$, then $A$ is closed upwards (downwards).

(ii) If $A_{i} \in \mathscr{F}$ for all $i=1, \ldots, n$, then $A \in \mathscr{F}$.

Proof. (a) Trivial.

(b) First let $A \in \mathscr{F}$. Obviously, $-\infty, \infty \in A$ and $A$ is closed. Let $a \in A \backslash\{-\infty\}$. If $a \notin \lim (A)$, there is a $b \in \bar{\Omega}$ with $b<a$ and $(b, a) \subseteq A^{c}$. But by Remark $3.10 A^{c}$ is a disjoint union of open intervals of countable coterminality, and $a$ is a right endpoint of such an interval. Hence $\operatorname{cof}(a)=\boldsymbol{\aleph}_{0}$. Conversely, let $A$ satisfy the condition. Then $A^{c} \subseteq \bar{\Omega}$ is open and hence a disjoint union of open intervals. Let $a \in A$ be a (e.g. right) endpoint of such an interval. Then $a \notin \underset{\rightarrow}{\lim }(A)$ implies $\operatorname{cof}(a)=\boldsymbol{\aleph}_{0}$, hence the intervals have countable coterminality, and $A \in \mathscr{F}$ by Remark 3.10.

(c) (i) Trivial.

(ii) Without loss of generality let $I=\mathbf{N}$ and $a \in \bar{\Omega} \cup\{\infty\}$ with $\operatorname{cof}(a) \neq \boldsymbol{\aleph}_{0}$ and $a \in \lim \left(A_{i}\right)$ for each $i \in I$. We apply a standard argument of set theory. Let $n_{1}, n_{2}, n_{3}, \ldots$ be an arbitrary sequence in $\mathbf{N}$ which contains each positive integer infinitely many times. For any $b \in \bar{\Omega}$ with $b<a$ choose a sequence $b<b_{1}<b_{2}<$ $\ldots<a$ in $\bar{\Omega}$ with $b_{i} \in A_{n_{i}}$ for each $i \in I$, and put $b^{+}=\sup \left\{b_{i} \mid i \in I\right\}$. Then $b^{+}<a$ because $\operatorname{cof}(a) \neq \boldsymbol{\aleph}_{0}$, and $b^{+}=\sup \left\{b_{j} \mid j \in I, n_{j}=i\right\} \in A_{i}$ for each $i \in I$, hence $b^{+} \in A$ and $b<b^{+}<a$. This shows $a \in \lim (A)$.

(iii) Immediately by (c)(i), (ii), and (b).

(d)(i) is obvious, and (ii) follows from (d)(i) and (b).

Lemma 3.17. Let $A, B \in \mathscr{F}$ and $C=C_{1} \cup C_{2}$, where $C_{1}=(R(A) \cup S(A)) \cap$ $(R(B) \cup S(B))$ and $C_{2}=(L(A) \cup S(A)) \cap(L(B) \cup S(B))$.

(a) $R(A) \cup S(A), C_{1}$ and $C$ are closed upwards, and $L(A) \cup S(A), C_{2}$ and $C$ are closed downwards. $C$ is closed.

(b) $R(A) \cup S(A)\left(C_{1}\right)$ is cofinal to each $a \in R(A) \cup S(A)\left(a \in C_{1}\right)$ with $a \neq-\infty$ and $\operatorname{cof}(a) \neq \boldsymbol{\aleph}_{0} . L(A) \cup S(A)\left(C_{2}\right)$ is coinitial to each $a \in L(A) \cup S(A)\left(a \in C_{2}\right)$ with $a \neq \infty$ and $\operatorname{coi}(a) \neq \boldsymbol{\aleph}_{0}$.

(c) If $\operatorname{cof}(\Omega) \neq \aleph_{0}$ and $\infty \in S(A)$, then $R(A) \cup S(A)$ is closed upwards and cofinal. If $\operatorname{coi}(\Omega) \neq \boldsymbol{\aleph}_{0}$ and $-\infty \in S(A)$, then $L(A) \cup S(A)$ is closed downwards and coinitial.

Proof. (a) To demonstrate that $C_{1}$ and $C$ are closed upwards it suffices to show $\lim (C) \subseteq C_{1}$. Let $a \in \lim (C)$. Then $a \in A \cap B$ since $C \subseteq A \cap B$. If $a \in L(A) \cup$ $\overrightarrow{I(A)}$, there exists $b \in \vec{A}$ with $b<a$ and $[b, a) \subseteq I(A)$, hence $[b, a) \cap C=\varnothing$, a 
contradiction. This shows $a \in R(A) \cup S(A)$ and, by symmetry, $a \in C_{1}$. By letting $A=B$ it follows that $R(A) \cup S(A)$ is closed upwards.

(b) Let $-\infty \neq a \in R(A) \cup S(A)$ and $\operatorname{cof}(a) \neq \aleph_{0}$. There exists a set $B \subseteq A^{c}$ with $a=\sup B$. Choose $b \in B$. By Proposition 3.16(b) we obtain $a \in \lim _{\rightarrow}(A)$, hence there exists $b^{\prime}=\inf \{c \in A \mid b<c<a\}$. It follows that $b^{\prime} \in A \backslash(L(\vec{A}) \cup I(A))=$ $R(A) \cup S(A)$ and $b<b^{\prime}<a$. This shows $a \in \lim _{\rightarrow}(R(A) \cup S(A))$.

(c) Immediate from (a) and (b).

LEMmA 3.18. Let $A, B, F \in \mathscr{F}$ with $S(A) \cap F \subseteq S(B)$. Then there exists a set $U \in \mathscr{F}$ with $S(A) \cap U \subseteq S(B), R(A) \cap U \subseteq R(B) \cup S(B)$ and $L(A) \cap U \subseteq L(B)$ $\cup S(B)$.

Proof. For every $a \in R(A)$ let $a^{\prime}=\sup \{x \in A \mid a<x,[a, x] \subseteq A\} \in L(A)$. This defines an order-isomorphism ': $R(A) \rightarrow L(A)$ with $a<a^{\prime}$ and $\left[a, a^{\prime}\right] \subseteq A$ for each $a \in R(A)$. For $a \in R(A)$ choose elements $a_{1}, a_{2} \in A$ by Remark 2.3 with $a<a_{1}<$ $a_{2}<a^{\prime}$ and $\operatorname{coi}\left(a_{1}\right)=\operatorname{cof}\left(a_{2}\right)=\boldsymbol{\aleph}_{0}$. If $b \in L(A)$ satisfies $b=a^{\prime}$ for $a \in R(A)$, we also write $b_{2}=a_{2}$. Let $C_{1}=(R(A) \cup S(A)) \cap(R(B) \cup S(B)), C_{2}=(L(A) \cup$ $S(A)) \cap(L(B) \cup S(B))$, and $C=C_{1} \cup C_{2}$. We put $T=C \cup D$ where

$$
D=\bigcup_{a \in R(A) \cap C}\left[a, a_{1}\right] \dot{\cup} \bigcup_{b \in L(A) \cap C}\left[b_{2}, b\right] .
$$

First let us show that $T$ is closed upwards in $\hat{\Omega}$. By Lemma 3.17, $C$ is closed. Hence, according to Proposition 3.16(d), it suffices to prove that $\lim _{\rightarrow}(D) \subseteq T$. Let $s \in \bar{\Omega} \cup\{\infty\}$ and $X \subseteq D$ with $s \notin X$ and $s=\sup X$. Now if there are $a \in R(A) \cap C$, $b \in L(A) \cap C$ and $x \in X$ with $y \in\left[a, a_{1}\right] \cup\left[b_{2}, b\right]$ for all $y \in X$ with $x<y$, we immediately get $x \in\left[a, a_{1}\right] \cup\left[b_{2}, b\right]$. Otherwise, to each $x \in X$ there exists an $a_{x} \in(R(A) \cap C) \cup(L(A) \cap C) \subseteq D$ with $x<a_{x}<s$, and we obtain a set $Y \subseteq$ $(R(A) \cap C) \cup(L(A) \cap C)$ with $s \notin Y$ and $s=\sup Y$. Now $Y \subseteq C$ implies $s \in C$, since $C$ is closed, proving $\lim (D) \subseteq T$.

By a symmetrical argument, $T$ is closed downwards and hence closed. Thus $T \cap F$ is closed and $-\infty, \infty \in T \cap F$, and now we show $T \cap F \in \mathscr{F}$ by Proposition 3.16(b). Let $c \in T \cap F$ with $c \neq-\infty$ and $\operatorname{cof}(c) \neq \boldsymbol{\aleph}_{0}$. We claim $c \in \lim _{\rightarrow}(T \cap F)$. First observe $c \neq b_{2}$ for all $b \in L(A) \cap C$ since $\operatorname{cof}\left(b_{2}\right) \doteq \boldsymbol{\aleph}_{0}$ by construction. Next assume $c \in\left(a, a_{1}\right]\left(c \in\left(b_{2}, b\right]\right)$ for some $a \in R(A) \cap C(b \in L(A) \cap C)$. Since $F \in \mathscr{F}$ is cofinal to $c \in F,(a, c] \cap F\left(\left(b_{2}, c\right] \cap F\right)$ is also cofinal to $c$, proving $c$ $\in \lim (T \cap F)$. Hence now we can assume $c \in C$ and $c \notin L(A) \cap C$, thus $c \in$ $(R(\vec{A}) \cup S(A)) \cap C \cap F$. Observe $R(A) \cap C \subseteq R(B) \cup S(B)$ and $S(A) \cap F \subseteq$ $S(B)$ by assumption, hence $c \in R(B) \cup S(B)$ and so $c \in C_{1}$. By Lemma 3.17(b) and Proposition 3.16(c) we get $c \in \lim _{\rightarrow}\left(C_{1}\right)$ and $c \in \lim _{\rightarrow}\left(C_{1} \cap F\right)$, thus $c \in$ $\lim (T \cap F)$. Together with a symmetrical argument, this shows $T \cap F \in \mathscr{F}$.

Now put $U=T \cap F \in \mathscr{F}$. Then $S(A) \cap U \subseteq S(A) \cap F \subseteq S(B)$ by assumption, and also $R(A) \cap U \subseteq R(A) \cap T=R(A) \cap C \subseteq R(B) \cup S(B)$ and $L(A) \cap U \subseteq$ $L(B) \cup S(B)$.

Now we can simplify Theorem 3.12 . 
THEOREM 3.19. Let $\Omega$ be a doubly homogeneous chain, and let $\alpha, \beta \in A(\Omega)$ and $A=F(\alpha), B=F(\beta)$. The following are equivalent.

(1) $(\alpha) \subseteq(\beta)$.

(2) There exist $F \in \mathscr{F}$ with $F \subseteq A \cap B$ and the following properties:

(i) $S(A) \cap F \subseteq S(B)$;

(ii) whenever $a, b \in F$ satisfy $a<b$ and $[a, b] \subseteq B$, then $[a, b] \subseteq A$.

Proof. (1) $\rightarrow$ (2): By Theorem 3.12.

(2) $\rightarrow$ (1): By Lemma 3.18 and Theorem 3.12.

Definition 3.20 of A PARTIALly ORDERED SET $(\mathscr{S}(\Omega), \leqslant)$. Let $A, B \in \mathscr{F}=\mathscr{F}(\Omega)$. If condition (2) of Theorem 3.19 is satisfied, we put $A \leqslant B$. Then the relation $\leqslant$ is reflexive and transitive on $\mathscr{F}$. Put $A \sim B$ if $A \leqslant B$ and $B \leqslant A(A, B \in \mathscr{F})$. Then for any $A \in \mathscr{F}$ let $[A]=\{B \in \mathscr{F} \mid A \sim B\}$ designate the equivalence class of $A$ in $\mathscr{F}$ with respect to $\sim$, and let $\mathscr{S}(\Omega)=\{[A] \mid A \in \mathscr{F}\}=\mathscr{F} / \sim$ designate the factor space. For $A, B \in \mathscr{F}$ let $[A] \leqslant[B]$ iff $A \leqslant B$. Then $(\mathscr{S}(\Omega), \leqslant)$ is a partially ordered set.

Now Theorem 3.19 and Proposition 3.3 immediately imply our characterization of $\left(\mathscr{N}_{1}(A(\Omega)), \subseteq\right)$.

THEOREM 3.21. Let $\Omega$ be a doubly homogeneous chain. Then the map $\phi: \mathscr{N}_{1}(A(\Omega))$ $\rightarrow \mathscr{S}(\Omega)$ defined by $(\alpha)^{\phi}=[F(\alpha)]$ is an isomorphism from the partially ordered set $\left(\mathscr{N}_{1}(A(\Omega)), \subseteq\right)$ onto $(\mathscr{S}(\Omega), \leqslant)$. Hence $(\mathscr{S}(\Omega), \leqslant)$ is a join semilattice, and $[A] \vee$ $[B]=[A \cap B]$ for all $A, B \in \mathscr{F}(\Omega) .(\mathscr{N}(A(\Omega)), \subseteq)$ is isomorphic to the set of all ideals of $(\mathscr{S}(\Omega), \leqslant)$.

Note that because of Remark 3.10 or Proposition 3.16(b), the set $(\mathscr{S}(\Omega), \leqslant)$ has been defined completely within $(\bar{\Omega}, \leqslant)$ without reference to $A(\Omega)$. In particular, by Theorem 3.21 the structure of $(\mathscr{N}(A(\Omega)), \subseteq)$ depends only on the structure of $(\bar{\Omega}, \leqslant)$. Thus we have

Corollary 3.22. Let $\Omega_{1}, \Omega_{2}$ be two doubly homogeneous chains with isomorphic Dedekind completions $\bar{\Omega}_{1} \cong \bar{\Omega}_{2}$. Then $A\left(\Omega_{1}\right)$ and $A\left(\Omega_{2}\right)$ have isomorphic normal subgroup lattices.

However, in the situation of Corollary 3.22 it is possible that the groups $A\left(\Omega_{1}\right)$ and $A\left(\Omega_{2}\right)$ are not isomorphic and, moreover, do not even satisfy the same sentences of group theory. For example, take $\Omega_{1}=\mathbf{Q}$ and $\Omega_{2}=\mathbf{R}[11,13,14]$.

EXAMPLE 3.23. Let $\omega_{1}$ be the least uncountable ordinal with smallest element 0 and $I_{0}=[0,1) \subseteq \mathbf{R}, I_{1}=I_{0} \backslash \mathbf{Q}, I_{2}=I_{0} \cap \mathbf{Q}$. For $i=1,2,3$ let $\mathbf{L}_{i}^{\prime}=I_{i} \times \omega_{1}$ ordered lexicographically: $\left(a_{1}, \mu_{1}\right)<\left(a_{2}, \mu_{2}\right)$ iff either $\mu_{1}<\mu_{2}$ or $\mu_{1}=\mu_{2}$ and $a_{1}<a_{2}$ $\left(a_{j} \in I_{i}, \mu_{j} \in \omega_{1}\right.$ for $\left.j=1,2\right)$. Let $\mathbf{L}_{i}=\mathbf{L}_{i}^{\prime} \backslash\{(0,0)\}$. Then each $\left(\mathbf{L}_{i}, \leqslant\right)$ is called a long line and is a doubly homogeneous chain with $\operatorname{cof}\left(\mathbf{L}_{i}\right)=\boldsymbol{\aleph}_{1}, \operatorname{coi}\left(\mathbf{L}_{i}\right)=\boldsymbol{\aleph}_{0}$ and $\overline{\mathbf{L}}_{i}=\mathbf{L}_{1}$. Hence the normal subgroup lattices $\mathcal{N}\left(A\left(\mathbf{L}_{i}\right)\right)(i=1,2,3)$ are pairwise isomorphic.

Using the results and methods of this section, in Droste and Shelah [9] a complete set-theoretic characterization and construction of the class of all normal subgroup lattices $\mathscr{N}(A(\Omega))$ ( $\Omega$ a doubly homogeneous chain) is given, without assuming, as in 
Theorem 3.21, the class of all doubly homogeneous chains to be given. As a consequence, an open problem in [6, p. 124], whether in Theorem 3.19 condition (2)(ii) is necessary if $\beta \neq$ id (if $\beta=\mathrm{id}$, it is clearly necessary; take any $\alpha \in$ $B(\Omega) \backslash\{$ id $\}$, has been answered positively. It was also shown in [9] that $\mathscr{N}_{1}(A(\Omega))$ is indeed closed under even countably infinite intersections; hence $(\mathscr{S}(\Omega), \leqslant)$ is a lattice closed under countable infima.

4. Group-theoretic properties of $A(\Omega)$. In this section we will use our characterization of $\mathscr{N}(A(\Omega))$ obtained in Theorems 3.19 and 3.21 to derive further grouptheoretical properties of $A(\Omega)$, in particular, to generalize our starting point, Theorem 3.1 .

LEMMA 4.1. (a) There exist $F_{i} \in \mathscr{F}(i=1,2,3)$ and $y, z \in \bar{\Omega}$ with

(i) $S\left(F_{1}\right)=F_{1}$;

(ii) $R\left(F_{2}\right)=\{-\infty\}, \infty \in S\left(F_{2}\right)=F_{2} \cap[y, \infty]$;

(iii) $L\left(F_{3}\right)=\{\infty\},-\infty \in S\left(F_{3}\right)=F_{3} \cap[-\infty, z]$.

(b) Whenever $F_{i} \in \mathscr{F}$ satisfy the conditions in (a) and $\alpha_{i} \in A(\Omega)$ are such that $F\left(\alpha_{i}\right)=F_{i}(i=1,2,3)$, then $\left(\alpha_{1}\right)=A(\Omega),\left(\alpha_{2}\right)=R(\Omega)$ and $\left(\alpha_{3}\right)=L(\Omega)$.

Proof. (a) Let $I=\left\{(a, b) \mid a, b \in \hat{\Omega}, a<b, \cot ((a, b))=\aleph_{0}\right\}$. By Zorn's lemma, there exists a maximal subset $M \subseteq I$ such that the elements of $M$ are pairwise disjoint. Let $F_{\sigma}=\dot{U}\{Z \mid Z \in M\}$ and $F_{1}=\hat{\Omega} \backslash F_{\sigma}$. Clearly $F_{1} \in \mathscr{F}$, and we claim $S\left(F_{1}\right)=F_{1}$. Otherwise, there are $a, b \in F_{1}$ with $a<b$ and $[a, b] \subseteq F_{1}$. Choose $c, d \in \bar{\Omega}$ with $a<c<d<b$ and $\cot (c, d)=\boldsymbol{\aleph}_{0}$. Then $M \dot{\cup}\{(c, d)\}$ provides a counterexample to the maximality of $M$. This shows (i).

Now choose $y \in F_{1}^{c}$ with coi $(y)=\boldsymbol{\aleph}_{0}$, and let $F_{2}=[-\infty, y] \cup F_{1}$. Then $F_{2} \in \mathscr{F}$ satisfies (ii). Analogously, we obtain (iii).

(b) First we prove $\left(\alpha_{1}\right)=A(\Omega)$. Choose any $\alpha \in A(\Omega)$. Obviously, $S(\alpha) \cap F_{1} \subseteq$ $S\left(F_{1}\right)$. By $S\left(F_{1}\right)=F_{1}$, there are no $a, b \in \bar{\Omega}$ with $a<b$ and $[a, b] \subseteq F_{1}$. Hence Theorem 3.19 implies $\alpha \in\left(\alpha_{1}\right)$.

Next we show $\left(\alpha_{2}\right)=R(\Omega)$. Clearly $\alpha_{2} \in R(\Omega)$ since $-\infty \in R\left(\alpha_{2}\right)$. Now let $\beta \in R(\Omega)$. Choose $c \in \Omega$ with $[-\infty, c] \subseteq F(\beta)$ and $\gamma \in A(\Omega)$ with $y<c^{\gamma}$. Then $[-\infty, y] \subseteq F\left(\beta^{\gamma}\right)$ and $S\left(\beta^{\gamma}\right) \cap F_{2} \subseteq(y, \infty] \cap F_{2} \subseteq S\left(\alpha_{2}\right)$. Whenever $d, e \in F_{2}$ satisfy $d<e$ and $[d, e] \subseteq F\left(\alpha_{2}\right)$, then $e \leqslant y$ and $[d, e] \subseteq F\left(\beta^{\gamma}\right)$. By Theorem 3.19 this shows $\beta \in\left(\alpha_{2}\right)$. It can be shown analogously that $\left(\alpha_{3}\right)=L(\Omega)$.

As an immediate consequence we obtain

THEOREM 4.2. Let $\Omega$ be a doubly homogeneous chain and $G$ any one of the groups $A(\Omega), R(\Omega)$, or $L(\Omega)$. Then:

(a) $G \in \mathscr{N}_{1}$.

(b) Each proper normal subgroup of $G$ is contained in a maximal proper normal subgroup of $G$.

(c) $\bigcap\left\{M \mid M r_{\max } G\right\}=\{\alpha \in G \mid \beta \in G,(\alpha) \cdot(\beta)=G$ imply $(\beta)=G\}$.

(d) Let $N_{1}, N_{2} \triangleleft G$ and $\alpha \in A(\Omega)$ such that $N_{1} \cdot N_{2}=G$ and $N_{1} \cap N_{2}=(\alpha)$.

Then $N_{1}, N_{2} \in \mathcal{N}_{1}$. 
Proof. (a) Immediate by Lemma 4.1.

(b) By (a) and Zorn's lemma.

(c) Straightforward using (a) and (b).

(d) By (a), $G=(\gamma)$ for some $\gamma \in A(\Omega)$. Choose $\beta_{i} \in N_{i}(i=1,2)$ such that $\gamma \in\left(\beta_{1}\right) \cdot\left(\beta_{2}\right)$. Then $G=\left(\beta_{1}\right) \cdot N_{2}$, and by the distributivity of $\mathcal{N}(A(\Omega))$ we obtain

$$
N_{1}=\left(\left(\beta_{1}\right) \cdot N_{1}\right) \cap\left(\left(\beta_{1}\right) \cdot N_{2}\right)=\left(\beta_{1}\right) \cdot\left(N_{1} \cap N_{2}\right)=\left(\beta_{1}\right) \cdot(\alpha) \in \mathscr{N}_{1} .
$$

All of the results established so far are obvious by Theorem 3.1 if $\cot (\Omega)=\boldsymbol{\aleph}_{0}$. Now we want to show that the structure of $(\mathscr{N}(A(\Omega)), \subseteq)$ is indeed much more complicated if $\operatorname{cof}(\Omega) \neq \boldsymbol{\aleph}_{0}$ or $\operatorname{coi}(\Omega) \neq \boldsymbol{\aleph}_{0}$. For this we need some preparations.

Notation 4.3. Let $\operatorname{cof}(\Omega) \neq \boldsymbol{\aleph}_{0}$. A set $T \subseteq \bar{\Omega}$ is called a good club if $T$ is closed and unbounded in $\bar{\Omega}$ and $(T, \leqslant)$ is well ordered, i.e. if

$$
\lim _{\leftarrow}(T)=\varnothing \quad \text { and } \quad \infty \in \lim _{\rightarrow}(T \cup\{\infty\}) \subseteq T \cup\{\infty\}
$$

Proposition 4.4. Let $A \subseteq \bar{\Omega}$.

(a) We have $\lim (\lim (A)) \subseteq \lim (A)$ and $\lim (\lim (A)) \subseteq \lim (A)$, and $\lim (A)$ is closed.

(b) If $a \in \lim (A)$ and $\operatorname{cof}(a) \neq \aleph_{0}$, then $a \in \lim (\lim (A))$.

(c) Let $\operatorname{cof}(\vec{\Omega}) \neq \boldsymbol{\aleph}_{0}$ and let $T \subseteq \bar{\Omega}$ be cofinal and such that $(T, \leqslant)$ is well ordered. Then $T^{\prime}=\lim (T) \cap \bar{\Omega}$ is a good club with $\operatorname{cof}(a)=\boldsymbol{\aleph}_{0}$ for each $a \in T^{\prime} \backslash \lim \left(T^{\prime}\right)$. Hence $T^{\prime}$ is cofinal to each $a \in T^{\prime}$ with $\operatorname{cof}(a) \neq \boldsymbol{\aleph}_{0}$.

(d) Let $\operatorname{cof}(\Omega) \neq \aleph_{0}, T \subseteq \bar{\Omega}$ a good club and $A$ closed above and cofinal. Then $T \cap A$ is a good club.

(e) If $\kappa=\operatorname{cof}(\Omega) \neq \aleph_{0}$, there exists a good club $T \subseteq \bar{\Omega}$ with $T \cong \kappa$.

Proof. (a) We only show $\lim _{\rightarrow}(\lim (A)) \subseteq \lim _{\rightarrow}(A)$. Let $a \in \lim _{\rightarrow}(\lim (A))$ and $B \subseteq \lim (A)$ with $a \notin B$ and $a=\sup B$. Choose $b \in B$. There exists $b^{\prime} \in B$ with $b<b^{\prime}<a$ and then, since $b^{\prime} \in \lim (A)$, an element $a^{\prime} \in A$ with $a^{\prime} \in\left(b, b^{\prime}\right) \cup$ $\left(b^{\prime}, a\right)$; in particular $b<a^{\prime}<a$. This shows $a \in \lim (A)$.

(b) Choose any $x \in \bar{\Omega}$ with $x<a$, and then a set $A^{\prime}=\left\{a_{i} \mid i \in \mathbf{N}\right\} \subseteq A$ with $x<a_{i}<a_{i+1}<a$ for all $i \in \mathbf{N}$. Let $y=\sup A^{\prime}$. Then $x<y<a$ by $\operatorname{cof}(a) \neq \boldsymbol{N}_{0}$, and $y \in \lim (A)$. This shows $a \in \lim (\lim (A))$.

(c) We apply (a) and (b). Obviously $\lim \left(T^{\prime}\right) \subseteq \lim (T)=\varnothing$ and $T^{\prime} \cup\{\infty\}=$ $\lim (T)$, hence $T^{\prime}$ is well ordered and closed in $\bar{\Omega}$. If $a \in T^{\prime} \cup\{\infty\}=\lim _{\rightarrow}(T)$ and $\operatorname{cof}(a) \neq \boldsymbol{\aleph}_{0}$, then

$$
a \in \lim _{\rightarrow}(\lim (T))=\lim _{\rightarrow}\left(T^{\prime} \cup\{\infty\}\right)=\lim _{\rightarrow}\left(T^{\prime}\right) \subseteq \lim \left(T^{\prime}\right)
$$

in particular $\infty \in \lim \left(T^{\prime}\right)$.

(d) By Proposition 3.16(c), $B=(T \dot{\cup}\{\infty\}) \cap A$ is closed above and cofinal, and as a subset of $T \cup\{\infty\}, B$ is well ordered. Hence $T \cap A$ is a good club.

(e) By standard set theory. 
The following construction will be used several times.

Construction 4.5. Assume $\operatorname{cof}(\Omega) \neq \boldsymbol{\aleph}_{0}$, and let $Z \subseteq \bar{\Omega}$ be a good club with $\operatorname{cof}(a)=\aleph_{0}$ for all $a \in Z \backslash \lim (Z)$ (cf. Proposition 4.4). For each $a \in Z$ let $a^{+}=$ $\min \{z \in Z \mid a<x\}$ designate the successor of $a$ in $Z$, and choose $a^{\prime} \in\left(a, a^{+}\right)$with $\operatorname{coi}\left(a^{\prime}\right)=\boldsymbol{\aleph}_{0}$. Furthermore, find $x \in \bar{\Omega}$ with $x<Z$ and $\operatorname{coi}(x)=\boldsymbol{\aleph}_{0}$. Then let $Z^{+}\left(x, \mathbf{a}^{\prime}\right)=[-\infty, x] \dot{\cup} \dot{U}_{a \in Z}\left[a, a^{\prime}\right] \dot{\cup}\{\infty\}$. Observe $\left(Z^{+}\left(x, \mathbf{a}^{\prime}\right)\right)^{c}=(x, \min Z)$ $\dot{\cup} \dot{U}_{a \in Z}\left(a^{\prime}, a^{+}\right)$, and the open intervals have countable coterminality by construction. Hence $Z^{+}\left(x, \mathbf{a}^{\prime}\right) \in \mathscr{F}$ by Remark 3.10.

The procedure used in the following proof is typical for our setting.

LEMmA 4.6. Let $\varepsilon \in R(\Omega) \backslash B(\Omega)$. Assume that there exists either an $F \in \mathscr{F}$ with $S(\varepsilon) \cap F=\{\infty\}$, or, if $\operatorname{cof}(\Omega) \neq \boldsymbol{\aleph}_{0}$, more generally, a closed and unbounded set $Z \subseteq \hat{\Omega}$ with $S(\varepsilon) \cap Z=\{\infty\}$. Then $\varepsilon \in(\alpha)$ for each $\alpha \in A(\Omega) \backslash L(\Omega)$.

Proof. Let $\alpha \in A(\Omega) \backslash L(\Omega)$. If $\operatorname{cof}(\Omega)=\boldsymbol{\aleph}_{0}$ then Theorem 3.1(d) and the distributivity of $\mathcal{N}(A(\Omega))$ imply $(\alpha)=((\alpha) \cap L(\Omega)) \cdot((\alpha) \cap R(\Omega))$. Now $(\alpha) \cap$ $R(\Omega) \subseteq B(\Omega)$ would imply $(\alpha) \subseteq L(\Omega)$, a contradiction. Hence $(\alpha) \cap R(\Omega)=R(\Omega)$ by Theorem 3.1(c), (e), proving $\varepsilon \in R(\Omega) \subseteq(\alpha)$. Therefore we can now assume $\operatorname{cof}(\Omega) \neq \aleph_{0}$. Let $Z \subseteq \hat{\Omega}$ be closed and cofinal with $S(\varepsilon) \cap Z \cap \bar{\Omega}=\varnothing$. Put $A=$ $F(\alpha), E=F(\varepsilon)$ and choose $x, y \in \bar{\Omega}$ with $x<y,(-\infty, x] \subseteq I(E),(x, y) \nsubseteq A$, and $\operatorname{coi}(x)=\aleph_{0}$. By Proposition 4.4(c) there exists a good club $T \subseteq \bar{\Omega}$ with $y<T$. Now since $\infty \in S(E) \cap S(A)$, Lemma 3.17(c) and Propositions 3.16(c), and 4.4(d) imply that $U=T \cap(R(E) \cup S(E)) \cap(R(A) \cup S(A)) \cap Z$ is again a good club. Let $V=\lim (U) \cap \bar{\Omega} \subseteq U$. By Proposition 4.4(c), $V$ is a good club with $\operatorname{cof}(a)=\boldsymbol{\aleph}_{0}$ for each $a \in V \backslash \lim (V)$. For each $a \in V$ let $a^{+}=\min \{v \in V \mid a<v\}$ be the successor of $a$ in $V$. By $a \in Z \cap \bar{\Omega}$ we have $a \notin S(E)$, but then $a \in U$ implies $a \in R(E)$, and hence we can choose an element $a^{\prime} \in\left(a, a^{+}\right)$with $\left(a, a^{\prime}\right] \subseteq I(E)$ and $\operatorname{coi}\left(a^{\prime}\right)=\aleph_{0}$. Now construct $V^{+}=V^{+}\left(x, \mathbf{a}^{\prime}\right) \in \mathscr{F}$ as in Construction 4.5. Since $-\infty \in R(E)$, we have $V^{+} \backslash\{\infty\} \subseteq R(E) \cup I(E)$ and hence $S(E) \cap V^{+}=\{\infty\} \subseteq S(A)$.

Now let $c, d \in V^{+}$with $c<d$ and $[c, d] \subseteq A$. Since $(x, y) \nsubseteq A$, we have either $[c, d] \subseteq[-\infty, x] \subseteq E$ or $c, d \in V^{+} \backslash[-\infty, x]$. In the latter case $\infty \in S(A)$ implies $d \neq \infty$, hence there are $a_{1}, a_{2} \in V$ with $c \in\left[a_{1}, a_{1}^{\prime}\right], d \in\left[a_{2}, a_{2}^{\prime}\right]$ and $a_{1} \leqslant a_{2}$. But $a_{1}<a_{2}$ implies $\left[a_{1}^{\prime}, a_{2}\right] \subseteq A$, contradicting $a_{2} \in V \subseteq U \subseteq R(A) \cup S(A)$. Hence $a_{1}=a_{2}$, and this yields $[c, d] \subseteq\left[a_{1}, a_{1}^{\prime}\right] \subseteq E$ by construction. Thus Theorem 3.19 implies $\varepsilon \in(\alpha)$.

A slight generalization of the preceding lemma is given in

LEMMA 4.7. Let $\alpha, \beta \in A(\Omega)$ and $F \in \mathscr{F}$ satisfy $B \neq \mathrm{id}, S(\alpha) \cap F \subseteq S(\beta)$ and $S(\alpha) \cap F \cap \bar{\Omega}=\varnothing$. Then $\alpha \in(\beta)$.

Proof. First assume $F(\alpha)=\{-\infty, \infty\}$. Then $\cot (\Omega)=\boldsymbol{\aleph}_{0}$ by Remark 3.10, and $\beta \in R(\Omega)$ implies $-\infty \notin S(\beta) \supseteq S(\alpha) \cap F$, hence $\alpha \in R(\Omega)$. Likewise $\beta \in L(\Omega)$ implies $\alpha \in L(\Omega)$. The result follows from Theorem 3.1.

Now assume there exists an element $a \in F(\alpha) \cap \bar{\Omega}$. Let $\Omega_{1}=\{x \in \Omega \mid x<a\}$, $\Omega_{2}=\{x \in \Omega \mid a<x\}$ and $\alpha_{i}=\left.\left.\alpha\right|_{\Omega_{i}} \oplus \mathrm{id}\right|_{\Omega \backslash \Omega_{i}}(i=1,2)$. Then $(\alpha)=\left(\alpha_{1}\right) \cdot\left(\alpha_{2}\right)$ and we will show $\left(\alpha_{2}\right) \subseteq(\beta)$; then $\left(\alpha_{1}\right) \subseteq(\beta)$ by symmetry. Indeed, if $\infty \in L\left(\alpha_{2}\right)$, we 
have $\alpha_{2} \in B(\Omega) \subseteq(\beta)$ because $\beta \neq$ id. On the other hand if $\infty \in S\left(\alpha_{2}\right)$ then our assumption yields $S\left(\alpha_{2}\right) \cap F=\{\infty\} \subseteq S(\beta)$, hence $\beta \notin L(\Omega)$. Now Lemma 4.6 implies $\left(\alpha_{2}\right) \subseteq(\beta)$.

Lemma 4.8. There are $A_{i} \in \mathscr{F}(i=1,2,3,4)$ with $S\left(A_{1}\right)=\{-\infty, \infty\}, S\left(A_{2}\right)=\{\infty\}$, $S\left(A_{3}\right)=\{-\infty\}, S\left(A_{4}\right)=\varnothing$ and $A_{4} \neq \hat{\Omega}$.

Proof. Choose $x, y \in \bar{\Omega}$ with $x<y$ and $\operatorname{coi}(x)=\operatorname{cof}(y)=\boldsymbol{\aleph}_{0}$. We put $A_{4}=$ $[-\infty, x] \dot{\cup}[y, \infty] \in \mathscr{F}$.

If $\operatorname{cof}(\Omega) \neq \boldsymbol{\aleph}_{0}$, let $T \subseteq(y, \infty)$ be a good club with $\operatorname{cof}(a)=\boldsymbol{\aleph}_{0}$ for each $a \in T \backslash \lim (T)$. For each $a \in T$ let $a^{+}=\min \{t \in T \mid a<t\}$ and $a^{\prime} \in\left(a, a^{+}\right)$such that $\operatorname{coi}\left(a^{\prime}\right)=\boldsymbol{\aleph}_{0}$. Let $A_{1}^{\prime}=\dot{\cup}_{a \in T}\left[a, a^{\prime}\right] \dot{\cup}\{\infty\}$.

If $\operatorname{coi}(\Omega) \neq \boldsymbol{\aleph}_{0}$, there is analogously a closed coinitial set $R \subseteq(-\infty, x)$ satisfying $\lim (R)=\varnothing$, i.e. $(R, \leqslant)$ is inversely well ordered, and $\operatorname{coi}(b)=\boldsymbol{\aleph}_{0}$ for each $\vec{b} \in R \backslash \lim (R)$. For each $b \in R$ let $b^{-}=\max \{r \in R \mid r<b\}$ and $b^{\prime} \in\left(b^{-}, b\right)$ with $\operatorname{cof}\left(b^{\prime}\right)=\boldsymbol{\aleph}_{0}$. We put $B_{1}^{\prime}=\{-\infty\} \dot{\cup}_{b \in R}\left[b^{\prime}, b\right]$.

First we assume $\operatorname{cof}(\Omega) \neq \boldsymbol{\aleph}_{0}$ and $\operatorname{coi}(\Omega) \neq \boldsymbol{\aleph}_{0}$. Let $A_{1}=B_{1}^{\prime} \dot{\cup} A_{1}^{\prime}, A_{2}=[-\infty, x]$ $\dot{\cup} A_{1}^{\prime}$ and $A_{3}=B_{1}^{\prime} \dot{\cup}[y, \infty]$. Then $A_{2}=T^{+}\left(x, \mathbf{a}^{\prime}\right) \in \mathscr{F}$ as in Construction 4.5, and analogously $A_{3} \in \mathscr{F}$. Hence $A_{1}=A_{2} \cap A_{3} \in \mathscr{F}$. We have $S\left(A_{1}\right)=\{-\infty, \infty\}$, $S\left(A_{2}\right)=\{\infty\}$ and $S\left(A_{3}\right)=\{-\infty\}$.

Next assume $\operatorname{cof}(\Omega) \neq \boldsymbol{\aleph}_{0}$ and $\operatorname{coi}(\Omega)=\boldsymbol{\aleph}_{0}$. We put $A_{1}=\{-\infty\} \dot{\cup} A_{1}^{\prime}, A_{2}=$ $[-\infty, x] \dot{\cup} A_{1}^{\prime}$ and $A_{3}=\{-\infty\} \dot{\cup}[y, \infty]$.

If $\operatorname{cof}(\Omega)=\boldsymbol{\aleph}_{0}$ and $\operatorname{coi}(\Omega) \neq \boldsymbol{\aleph}_{0}$, proceed in a symmetric way.

Finally, if $\operatorname{cof}(\Omega)=\operatorname{coi}(\Omega)=\boldsymbol{\aleph}_{0}$, let $A_{1}=\{-\infty, \infty\}, A_{2}=[-\infty, x] \dot{\cup}\{\infty\}$ and $A_{3}=\{-\infty\} \dot{\cup}[y, \infty]$.

In each of these cases, $A_{1}, A_{2}, A_{3} \in \mathscr{F}$ satisfy the assertion.

Now we generalize Theorem 3.1.

THEOREM 4.9. Let $\Omega$ be a doubly homogeneous chain. Then

(a) $R(\Omega) / B(\Omega)$ is simple if and only if $\operatorname{cof}(\Omega)=\boldsymbol{\aleph}_{0}$.

(b) $L(\Omega) / B(\Omega)$ is simple if and only if $\operatorname{coi}(\Omega)=\boldsymbol{\aleph}_{0}$.

(c) $B(\Omega), L(\Omega)$ and $R(\Omega)$ are the only nontrivial proper normal subgroups of $A(\Omega)$ if and only if $\cot (\Omega)=\boldsymbol{\aleph}_{0}$.

(d) There exist smallest normal subgroups $N_{1}, N_{2}, N_{3}$ of $A(\Omega)$ satisfying

$(+) \quad B(\Omega) \subsetneq N_{1} \subseteq R(\Omega), \quad B(\Omega) \subsetneq N_{2} \subseteq L(\Omega), \quad$ and $\quad N_{3} \nsubseteq R(\Omega) \cup L(\Omega)$,

respectively.

Here we have $N_{i}=\left(\varepsilon_{i}\right)$ for any $\varepsilon_{i} \in A(\Omega)(i=1,2,3)$ with $S\left(\varepsilon_{1}\right)=\{\infty\}, S\left(\varepsilon_{2}\right)=$ $\{-\infty\}$ and $S\left(\varepsilon_{3}\right)=\{-\infty, \infty\}$.

Proof. First we show (d). The existence of $\varepsilon_{i} \in A(\Omega)$ as prescribed was shown in Lemma 4.8. Obviously $N_{i}=\left(\varepsilon_{i}\right)(i=1,2,3)$ satisfy $(+)$. Now let $B(\Omega) \subsetneq N \triangleleft R(\Omega)$. Choose $\beta \in N \backslash B(\Omega)$. Then $S\left(\varepsilon_{1}\right)=\{\infty\} \subseteq S(\beta)$ and $S\left(\varepsilon_{1}\right) \cap \bar{\Omega}=\varnothing$, hence $\varepsilon_{1} \in$ $(\beta) \subseteq N$ by Lemma 4.7 , proving $N_{1} \subseteq N$. In a similar way, Lemma 4.7 yields the minimality of $N_{2}$ and $N_{3}$. 
The normal subgroup lattice of $A(\Omega)$

(1) $\operatorname{coi}(\Omega)=\operatorname{cof}(\Omega)=\boldsymbol{\aleph}_{0}$

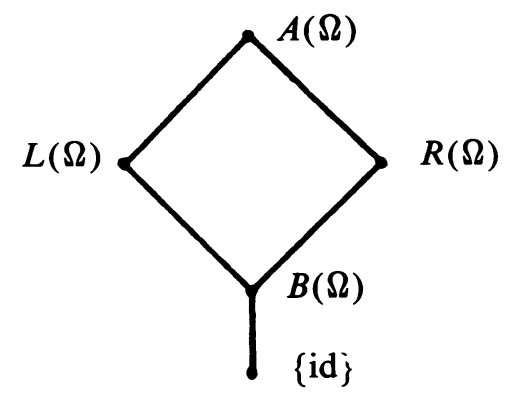

(2) $\operatorname{coi}(\Omega) \neq \aleph_{0}, \operatorname{cof}(\Omega)=\aleph_{0}$

(3) $\operatorname{coi}(\Omega)=\boldsymbol{\aleph}_{0}, \operatorname{cof}(\Omega) \neq \boldsymbol{\aleph}_{0}$
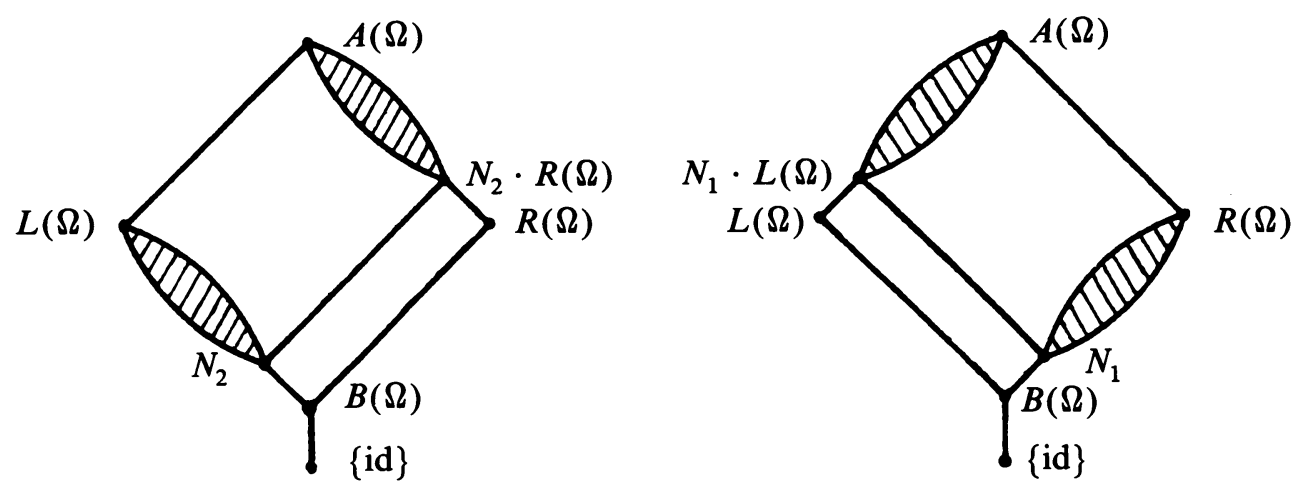

(4) $\operatorname{coi}(\Omega) \neq \aleph_{0}, \operatorname{cof}(\Omega) \neq \aleph_{0}$

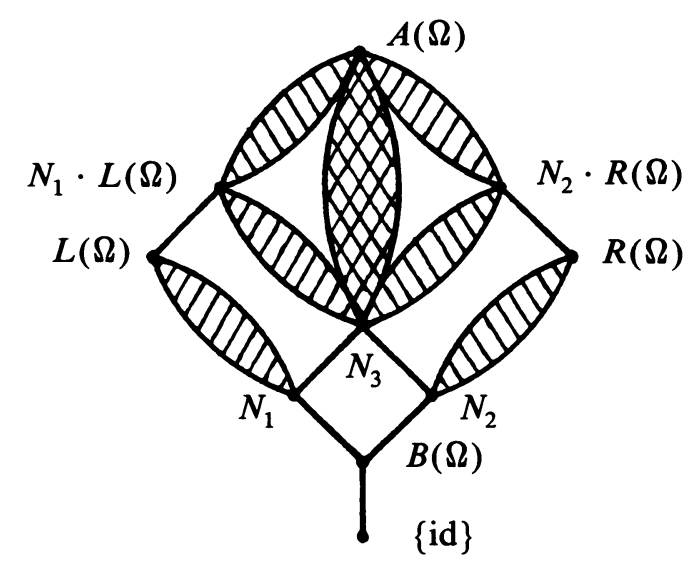


(a) Because of Theorem 3.1, we may assume $\operatorname{cof}(\Omega) \neq \boldsymbol{\aleph}_{0}$. We claim $B(\Omega) \subsetneq N_{1} \subsetneq$ $R(\Omega)$. Indeed, assume $\left(\varepsilon_{1}\right)=N_{1}=R(\Omega)$. According to Lemma 4.1, there are $A \in \mathscr{F}$, $\alpha \in A(\Omega), y \in \bar{\Omega}$ with $A=F(\alpha), R(A)=\{-\infty\}$ and $\infty \in S(A)=A \cap[y, \infty]$. Now by $\alpha \in R(\Omega)=\left(\varepsilon_{1}\right)$ and Theorem 3.19 there exists $F \in \mathscr{F}$ with $S(A) \cap F \subseteq$ $F\left(\varepsilon_{1}\right)=\{\infty\}$. However, by $\operatorname{cof}(\Omega) \neq \boldsymbol{\aleph}_{0}$ and Proposition 3.16(c), $S(A) \cap F=A \cap$ $F \cap[y, \infty]$ is cofinal in $\hat{\Omega}$. Hence $S(A) \cap F \cap \bar{\Omega} \neq \varnothing$, a contradiction.

(b) Analogously to (a).

(c) By (a) and (b).

The various possibilities for the structure of $(\mathscr{N}(A(\Omega)), \subseteq)$, as a consequence of Theorem 4.9, are depicted in the following diagram.

Because of the symmetry of this diagram and the distributivity of $\mathscr{N}(A(\Omega))$, we now turn to an examination of

$$
\mathscr{R}_{1}=\mathscr{N}_{1}(R(\Omega)) \backslash\{\mathrm{id}, B(\Omega)\}=\{(\alpha) \mid \alpha \in R(\Omega) \backslash B(\Omega)\}
$$

under the assumption $\kappa=\operatorname{cof}(\Omega) \neq \boldsymbol{\aleph}_{0}$. So far by Theorem 4.9(a), (d) we know that then $\left|\mathscr{R}_{1}\right| \geqslant 2$ (in fact, we even have $\left|\mathscr{R}_{1}\right| \geqslant 2^{\kappa}$, see $\left.[6,7]\right)$ and $\left(\mathscr{R}_{1}, \subseteq\right.$ ) has a smallest and a largest element. We wish to characterize when $\left(\mathscr{R}_{1}, \subseteq\right)$ is a Boolean algebra.

Definition 4.10 (CF. JeCH [15]). Let $\kappa \neq \aleph_{0}$ be a regular cardinal. We put $I(\kappa)=\{A \subseteq \kappa \mid \kappa \backslash A$ contains a closed unbounded subset of $\kappa\}$, the ideal of all thin subsets of $\kappa$. Then $A(\kappa)=P(\kappa) / I(\kappa)$, a Boolean algebra.

THEOREM 4.11. Let $\Omega$ be a doubly homogeneous chain with $\kappa=\operatorname{cof}(\Omega) \neq \aleph_{0}$. Then the following are equivalent:

(1) There exists a closed and cofinal subset $A \subseteq \bar{\Omega}$ with coi $(a)=\boldsymbol{\aleph}_{0}$ for each $a \in A$.

(2) $\left(\mathscr{R}_{1}, \subseteq\right)$ is a Boolean algebra.

(3) $\left(\mathscr{R}_{1}, \subseteq\right)$ is isomorphic to $A(\kappa)$.

For a proof of this result see [2] (where (1) and $\kappa=\boldsymbol{\aleph}_{1}$ is assumed) or [6, 7].

Finally, we note the following surprising consequence of Theorem 4.11, where ZFC denotes the standard system of axioms of set theory (including the axiom of choice) and $\kappa^{+}$the successor cardinal of the cardinal $\kappa$.

COROLlary 4.12. Let $\Omega=\mathbf{L}_{0}$, a long line, and $\boldsymbol{\aleph}$ the smallest cardinal number such that there exist no set $\left\{\alpha_{i} \mid i<\boldsymbol{\aleph}\right\} \subseteq R(\Omega)$ with the properties

(i) $\left(\alpha_{i}\right) \neq R(\Omega)$ for all $i<\boldsymbol{\kappa}$;

(ii) $\left(\alpha_{i}\right) \cdot\left(\alpha_{j}\right)=R(\Omega)$ for all $i, j<\boldsymbol{\aleph}, i \neq j$.

Then the question of whether $\boldsymbol{\aleph}=\left(2^{\aleph_{1}}\right)^{+}$is undecidable in $Z F C$.

Proof. If a system $\left\{\alpha_{i} \mid i<\boldsymbol{N}\right\}$ satisfies conditions (i) and (ii), then $\alpha_{i} \notin B(\Omega)$, hence $\left(\alpha_{i}\right) \in \mathscr{R}_{1}$ for all $i<\aleph$. By Theorem 4.11, $\left(\mathscr{R}_{1}, \subseteq\right)$ is isomorphic to $A\left(\boldsymbol{\aleph}_{1}\right)$. Hence $\boldsymbol{\aleph}$ is the smallest cardinal such that there exists no set $A \subseteq A\left(\boldsymbol{\aleph}_{1}\right)$ with $|A|=\aleph$ and $a \neq 1, a \vee b=1$ for all $a, b \in A, a \neq b$, where 1 denotes the largest element of $A\left(\boldsymbol{\aleph}_{1}\right)$. Now the undecidability result follows from Jech [15, pp. 434-436].

5. Lattice-theoretic properties of $A(\Omega)$. Here we will consider $A(\Omega)$ as an l-group again, and we will deal with minimal prime $l$-subgroups of $A(\Omega)$. Recall that an $l$-subgroup $P$ of an $l$-group $G$ is called prime if $P$ is convex and $f, g \in G, f \wedge g \in P$ always imply $f \in P$ or $g \in P$. 
Proposition 5.1. Let $G$ be an l-group, $C \subseteq G$ a convex l-subgroup, and $\phi \neq T \subseteq G$ a lower directed set of positive elements of $G$ such that $C \cap T=\varnothing$.

(a) [3, Theorem 2.2]. If $Q \subseteq G$ is maximal among those convex l-subgroups of $G$ containing $C$ which are disjoint from $T$, then $Q$ is prime.

(b) There exists a prime $P \supseteq C$ which is disjoint from $T$ and minimal in the set of all primes containing $C$.

Proof. (b) By Zorn's lemma and (a), there exists a prime $Q \supseteq C$ which is disjoint from $T$, and hence, again by Zorn's lemma, a minimal prime $P$ with $Q \supseteq P \supseteq C$.

LEMMA 5.2. Let $P$ be a prime and $C$ a convex l-subgroup of an arbitrary l-group $G$ such that $P \supseteq C$. The following are equivalent.

(1) $P$ is minimal among those primes containing $C$.

(2) For each $1<p \in P$ there exists an element $1<q \in G \backslash P$ with $p \wedge q \in C$.

Proof. (2) $\rightarrow$ (1): Let $Q$ be a prime with $C \subseteq Q \subseteq P$. Consider any $1<p \in P$ and find $1<q \in G \backslash P$ such that $p \wedge q \in C$. Then $p \wedge q \in Q, q \notin Q$, and $Q$ prime imply $p \in Q$, proving $P \subseteq Q$.

$\neg(2) \rightarrow \neg(1)$ : Suppose (2) is violated by $1<p \in P$ and let $T=\{p \wedge q \mid 1<q \in$ $G \backslash P\}$. Then $C \cap T=\varnothing$ and $T \neq \varnothing$. By Zorn's lemma and Proposition 5.1(a), there exists a prime $Q \supseteq C$ with $Q \cap T=\varnothing$. We claim $Q \subsetneq P$. Assume there exists $1<q \in Q \backslash P$. Then $p \wedge q \in T \cap Q$ contradicting $T \cap Q=\varnothing$. Hence $Q \subseteq P$, and $Q \neq P$ follows from $\phi \neq T \subseteq P$ and again $T \cap Q=\varnothing$.

As a consequence we obtain

CoRollary 5.3. Suppose $G$ is an l-group in which every polar is principal, and let $P$ be a prime of $G$. Then $P$ is a minimal prime of $G$ iff $p^{\perp \perp} \neq G$ for all $1<p \in P$. In particular, if $\Omega$ is a doubly homogeneous chain, a prime $P$ of $A(\Omega)$ is minimal iff $I(p) \neq \varnothing$ for all $p \in P$.

Proof. If $P$ is a nonminimal prime of $G$, then by the preceding lemma there is some $1<p \in P$ such that $p^{\perp} \subseteq P$. Now if $q \in G$ satisfies $p^{\perp}=q^{\perp \perp}$, then $q \in p^{\perp}$ and hence $p \vee q \in P$ and $(p \vee q)^{\perp \perp}=p^{\perp \perp} \vee p^{\perp}=G$. Conversely, if $P$ is a minimal prime of $G$ and $1<p \in P$, again by Lemma 5.2, we obtain $p^{\perp} \neq\{1\}$, i.e. $p^{\perp \perp} \neq G$. The final statement of the corollary now follows from the previous one and the following remark.

REMARK 5.4. Let $\Omega$ be a doubly homogeneous chain.

(a) Whenever id $<\alpha \in A(\Omega)$, then $\alpha^{\perp}=\{$ id $\}$ iff $I(\alpha)=\varnothing$.

(b) In $A(\Omega)$ all polars are principal.

Proof. (a) If $I(\alpha)=\varnothing$ and $\beta \in A(\Omega)$ satisfies $\alpha \wedge|\beta|=$ id, then $\bar{\Omega}=$ $I(\alpha \wedge|\beta|)=I(F(\alpha) \cup F(\beta))=I(\beta)$, where the last equality holds since $I(\alpha)=\varnothing$ and $I(\beta)$ is regular open, hence $\beta=$ id. Conversely, assume there are $a, b \in \hat{\Omega}$ with $a<b$ and $[a, b] \subseteq F(\alpha)$. If we define id $<\beta \in A(\Omega)$ such that $F(\beta) \supseteq[-\infty, a]$ $\dot{\cup}[b, \infty]$, we obtain $\beta \in \alpha^{\perp}$.

(b) Suppose $Q$ is a polar of $A(\Omega)$. By Zorn's lemma find a maximal set of pairwise disjoint positive elements $\left\{q_{i} \mid i \in I\right\} \subseteq Q$. For each $i \in I$ let $r_{i}$ designate the restriction of $q_{i}$ to $\hat{\Omega} \backslash F\left(q_{i}\right)$, and let $q$ be $\left.\oplus_{i \in I} r_{i} \oplus \mathrm{id}\right|_{A}$, where $A=\bigcap_{i \in I} F\left(q_{i}\right)$. Now 
$A=F(q)$, and $\hat{\Omega} \backslash A \subseteq B=\dot{\cup}_{q \in Q} \hat{\Omega} \backslash F(q)$, so $q^{\perp} \supseteq Q^{\perp}$. On the other hand $\hat{\Omega} \backslash A$ is dense in $B$, hence $q^{\perp} \subseteq Q^{\perp}$. We conclude $q^{\perp \perp}=Q$.

Now we state the main result of this section.

THEOREM 5.5. Let $\Omega$ be a doubly homogeneous chain and $N$ a proper normal subgroup of $A(\Omega)$. Then any prime $P$ minimal in the set of primes containing $N$ is minimal also in the set of all primes of $A(\Omega)$.

Proof. Suppose $P$ is minimal in the set of primes containing $N$, and for contradiction suppose $P$ contains $p>1$ with $I(p)=\varnothing$ (cf. Corollary 5.3). By Lemma 5.2, find $1<q \in A(\Omega) \backslash P$ such that $p \wedge q \in N$. Now $I(p \wedge q)=I(q)$ as in the proof of Remark 5.4(a). Hence $q \in N$ by Corollary 3.14, a contradiction.

COROLlaRY 5.6. Let $\Omega$ be a doubly homogeneous chain. Then every proper normal subgroup $N$ of $A(\Omega)$ is an intersection of minimal primes.

Proof. By Proposition 5.1(b) we have $N=\left\{P_{g} \mid g \in A(\Omega) \backslash N\right\}$, where $P_{g}$ is a prime minimal in the set of primes containing $N$ such that $g \notin P_{g}$. By Theorem 5.5, each $P_{g}$ is a minimal prime of $A(\Omega)$.

\section{REFERENCES}

1. R. D. Anderson, The algebraic simplicity of certain groups of homeomorphisms, Amer. J. Math. 80 (1958), 955-963.

2. R. N. Ball, Full convex l-subgroups of a lattice-ordered group, Ph.D. Thesis, University of Wisconsin, Madison, Wisc., 1974.

3. Math. 61 (1975), 7-16.

4. E. A. Bertram, On a theorem of Schreier and Ulam for permutations, J. Algebra 24 (1973), 316-322.

5. G. Birkhoff, Lattice theory, 3rd ed., Amer. Math. Soc. Colloq. Publ., vol. 25, Providence, R. I., 1973.

6. M. Droste, Wechselwirkung des algebraischen Struktur transitiver Automorphismengruppen mit der Geometrie ihres Operationsbereiches, Dissertation, Universität Essen, Essen, 1982.

7. The normal subgroup lattice of 2-transitive automorphism groups of linearly ordered sets (to appear).

8. M. Droste and R. Göbel, On a theorem of Baer, Schreier, and Ulam for permutations, J. Algebra 58 (1979), 282-290.

9. M. Droste and S. Shelah, A construction of all normal subgroup lattices of 2-transitive automorphism groups of linearly ordered sets, Israel J. Math. (to appear).

10. A. M. W. Glass, Ordered permutation groups, London Math. Soc. Lecture Note Series, vol. 55, Cambridge, 1981.

11. Y. Gurevich and W. C. Holland, Recognizing the real line, Trans. Amer. Math. Soc. 265 (1981), $527-536$.

12. G. Higman, On infinite simple groups, Publ. Math. Debrecen 3 (1954), 221-226.

13. W. C. Holland, The lattice-ordered group of automorphisms of an ordered set, Michigan Math. J. 10 (1963), 399-408.

14. M. Jambu-Giraudet, Bi-interpretable groups and lattices, Trans. Amer. Math. Soc. 278 (1983), 253-269.

15. T. Jech, Set theory, Pure and applied mathematics, Academic Press, New York, 1978.

16. J. T. Lloyd, Lattice ordered groups and o-permutation groups, Ph.D. Thesis, Tulane University, New Orleans, 1964.

17. E. B. Rabinovič and V. Z. Feinberg, Normal divisors of a 2-transitive group of automorphisms of a linearly ordered set, Mat. USSR Sb. 22 (1974), 187-200.

Department of Mathematics, Boise State University, Boise, Idaho 83725

Fachbereich 6 - Mathematik, Universität EsSEN, 4300 ESSEN 1, West Germany 